\title{
Metaphysics, Science, and Religion: A Response to Hud Hudson
}

\author{
Natalja Deng \\ Yonsei University, Underwood International College
}

\section{Introduction}

I found this book interesting and rewarding, as well as a real pleasure to read. It's a sustained defense of a rather provocative thesis, using original arguments in metaphysics, epistemology, the philosophy of time, space, personal identity, and the philosophy of religion.

The rather provocative thesis (let's call it PT) can be put as follows. Everything science (including astronomy, physics, geology, paleoanthropology, genetics, and evolutionary biology) has found out can be reconciled with an extreme literalism about the religious doctrines of the Fall and Original Sin. The Genesis story of creation, human rebellion, and expulsion from paradise, understood as concerning a primal sin committed by Adam and Eve a few thousand years ago, can be held to be literally true. For all we've found out, this first human couple lived a carefree life in a garden a few thousand years ago, shortly after creation, and through their disobedience brought disease, suffering, and death onto humanity.

There's also a more general aim that's closely related. This is to show that many supposed conflicts between science and religion are, at root, conflicts between rival metaphysical views. Combined with the further claim that 'metaphysics is a crazy business', because all metaphysical views contain 'seemingly bizarre commitments', this implies that clear-cut refutation of religious doctrine by science is extremely rare. In our efforts to make sense of the world, we have to choose between equally unpalatable options - or at least, between options of which none is completely free of 'seemingly bizarre commitments'. To adjudicate apparent conflicts between religion and science, we're forced to make sense of the world in one of these ways. There can therefore be no clear victories.

Hudson rightly asks that a dismissal of PT be backed up by genuine engagement with the case made for it. In what follows, I describe what I take the case for PT to be, and lay out my reasons for being unpersuaded by it; I also touch on the more general aim. My discussion is a partial response to the book, focusing on the case for PT.

\section{The case for PT}

Journal of Analytic Theology, Vol. 5, May 2017

10.12978/jat.2016-5.130000030406a

(C) 2017 Natalja Deng • (C) 2017 Journal of Analytic Theology 
The case for PT is contained in chapters 5-9. It turns on the claim that a particular way of thinking about the nature of space and time, the 'hypertime hypothesis' (HYP), is a live epistemic possibility. On HYP, while the past is in fact just as described by science, it used to be very different - or rather, it hyper-used to be very different. What is it for the past to hyper-have been different? Hudson's answer is as follows. It's for there to be a hyper-earlier hypertime at which our (or a relevantly similar) space-time block hyper-was hyper-present too, just like it hyper-is hyper-present at this hypertime. And at that hyper-earlier hypertime, it has a different past, namely one that includes a recent creation and Fall. To re-state: there is a hyper-succession of hypertimes, at each of which there exists a space-time block including all of world history, but world history differs from hypertime to hypertime. It hyper-is just as science describes, but hyper-was such as to include a recent creation and Fall.

So, actually, HYP isn't merely a particular way of thinking about the nature of space and time, i.e. a particular metaphysical view. It doesn't just say that there is a hyper-succession of hypertimes, each of which hosts a (or the one) space-time block. It adds to this a specific further claim about the contents of these space-time blocks (or of our space-time block at different hypertimes), namely that this one, the hypercurrent one, has a past accurately described by the relevant sciences, while another one, a hyper-previous one, has a past accurately described by Genesis. That's how HYP is relevant to PT, and how securing that HYP is a live epistemic possibility would lend support to PT. Admittedly, the strategy is intended to be adaptable. The metaphysical view involving hypertime is intended to be combinable with various further claims about mismatched contents of space-time blocks. That way science can be made compatible with a host of apparently implausible claims about the past or indeed the future. But the present point takes this into account. The point is just that in each of these cases, what needs to be a live epistemic possibility is the metaphysical view plus some specific further claim(s) concerning the contents of different spacetime blocks.

So far, I've described a key element of the case for PT; now we have to ask what the case for that key element is. Why think HYP is a live epistemic possibility? At this point chapters 5-9 lend themselves to somewhat different interpretations, corresponding to different answers. One prominent interpretation, enjoying ample textual support, is that the answer is roughly, because HYP has not been conclusively ruled out. On an alternative interpretation, the answer is roughly, because HYP is no less plausible than some other metaphysical views that are live contenders, and because in addition, HYP can do useful work in the philosophy of religion (chapters 7 \& 8).

I'll discuss these in turn. But first, I want to acknowledge the possibility that distinguishing between them goes against the spirit of the proposal. Maybe there is a background assumption to the effect that all it takes to establish plausibility in metaphysics is to be able to steer clear of conclusive refutation. This would amount to an 'anything goes' kind of attitude that doesn't even recognize degrees of plausibility in metaphysics but simply welcomes all views as equally (im?)plausible as long as they can't be conclusively ruled out (which presumably would be quite a lot). 


\section{One interpretation of the case for PT}

Let's suppose that's not the idea. ${ }^{1}$ (After all, if there are no degrees of plausibility in metaphysics, why attempt to strengthen the case for any view by arguing that it can do useful work?) Then the first answer may instead be motivated by the following thought. All it takes to establish PT is to show that HYP has not been conclusively ruled out - not because that's all it takes for plausibility in metaphysics, but because that's all it takes for being a live epistemic possibility in the relevant sense.

Chapter 6 in particular lends support to this interpretation because it makes explicit that HYP is a kind of sceptical hypothesis. In some sense, it's on a par with Bertrand Russell's hypothesis that the world came into existence five minutes ago (complete with misleading evidence), or the hypothesis that you're a disembodied brain floating in a vat of nutrient fluids. Consider the following argument pattern:

(1) I do not know that I am not in a plenitudinous hypertime world.

(2) If I do not know that I am not in a plenitudinous hypertime world, then I do not know that certain pairs of propositions are inconsistent.

(3) So, I do not know that certain pairs of propositions are inconsistent.

Here, 'plenitudinous' is 'a stand-in for a rich-enough hypertime environment to provide the reconciling resources hinted at in (2)' (122). ${ }^{2}$ That is, (1) is about whichever hypothesis conjoins a metaphysical hypertime view with suitable claims about the contents of space-time blocks, such as that one includes the Fall and another one doesn't (so 'I do not know that HYP is false' is a candidate first premise).

The pattern is modelled on familiar sceptical arguments (where ' $p$ ' is e.g. I'm holding a phone):

(4) I do not know that I'm not a brain in a vat.

(5) If I do not know that I'm not a brain in a vat, then I do not know that p.

\footnotetext{
${ }^{1}$ Admittedly, on p. 15 Hudson says that ' $[t]$ he perennial debates in [metaphysics] are chock full of positions that maintain their place on the lists of candidate solutions not because the arguments in their favor are so powerful, but precisely because they have not been decisively eliminated and all the alternatives seem so outlandish'. Incidentally, this raises the question of whether Hudson thinks the positions should be so treated, and if not, how this lamentable state of affairs (if it obtains) can be cited in support of another 'crazy metaphysics'. (I return to the point below.) But in any case, the continuation of the quote suggests a weaker claim, namely that there are no views without costs: 'Once one is safely beyond engaging the hopeless project of unearthing the only metaphysics utterly free of seemingly bizarre commitments, the pleasures of constructing consistent packages and of discovering just which views can fit together and complement one another are there for the taking'. Presumably this process requires there to be degrees of plausibility, since (by Hudson's lights) some views fit together better than others.

${ }^{2}$ All page citations are from Hudson 2014.
} 
(6) So, I do not know that $p$.

At this point the sceptical move can seem surprisingly unambitious, unable to generate much support for PT. If no more is being claimed on behalf of HYP than on behalf of the brain-in-vat scenario, nothing very remarkable follows that resembles PT. Knowledge of the incompatibility of science with a literal Genesis story would then be as secure as knowledge that I'm holding a phone. PT promises more.

However, Hudson does go on to claim more on behalf of HYP than familiar sceptical scenarios, namely that various rejoinders to familiar sceptical arguments are more likely to succeed there than with HYP. For example, he has higher hopes for a Moorean shift in response to brains in vats than in response to HYP. (A Moorean shift turns the sceptical argument on its head, insisting that I know that p, and (5), hence I know I'm not a brain in a vat.) We may be able to know that we're holding phones, but we're not able to know that science and a literal Genesis story are incompatible.

One question about this is whether it's quite the right comparison. Hudson emphasizes our limited capacity for discerning metaphysical incompatibility. But suppose we consider the following argument:

(1) I do not know that HYP is false.

(2') If I do not know that HYP is false, then I do not know that the universe is older than a few thousand years. (If you think this betrays a misunderstanding of HYP, hold that thought.)

(3') So, I do not know that the universe is older than a few thousand years.

Is a Moorean shift in worse shape here than in the case of brains in vats? Not so clearly. Suppose I know I'm holding a phone and I can deduce that I know I'm not a brain in a vat. Now compare: I know the universe is older than a few thousand years, and I can deduce that I know HYP is false. No doubt one can raise worries about this latter Moorean argument, but it's not clearly in worse shape than the former. Certainly, judging that one knows that the universe is older than a few thousand years doesn't require one to make "judgements of mere metaphysical possibility about complicated and abstruse mixtures of science and religion" (p. 126). It's not "a dogmatic claim to metaphysical knowledge where intellectual humility is called for" (p. 124).

The point is related to an underlying dissimilarity between HYP and familiar sceptical scenarios. Recall that HYP is not just a metaphysical view of the nature of time and space involving multiple space-time blocks at different hypertimes. It's also a specific further claim about certain mis-matched contents of those blocks, where one block includes a literal Genesis past and the other includes a past as described by science. It doesn't just challenge our claim to knowledge of the metaphysical nature of space and time. It challenges our claim to knowledge about a specific range of claims about the past, such as that the universe is older than a few thousand years. And while that makes it bolder, it also makes it less distinctive qua sceptical scenario, 
and more easily responded to in a Moorean fashion. It's as if a brain in vat scenario were conjoined with the specific further claim that all phones have cracked screens.

But is this the right way to think about HYP (or does (2') betray a misunderstanding)? One might insist that HYP doesn't imply that it's not the case that the universe is older than a few thousand years. According to the hyper-present block, the universe is older than that. It's just that it hyper-was younger - where that doesn't just mean it used to be younger (though that's also true), but that a hyper-previous block is shorter (towards its past).

This is an important objection, because it gets us to the heart of the problem. I just said that there having hyper-been a shorter block isn't what makes it the case that the universe used to be younger. Instead, what makes that true is something about the hyper-present space-time block. And what there having hyper-been a shorter block makes true is that there hyper-was a shorter block. But now we have to ask, what does that mean, and why is it relevant? What is it about hypertime that earns it the label? The language is suggestive, and we're told in some ways hypertime is like an additional 'time-like' dimension. But strictly speaking, it's not a dimension, and there is an open question about what makes it 'time-like' (no relation to the term as used in relativity theory). Suppose I said that there is a copy of our (or a relevantly similar) space-time block at a hyper-schmevious hyper-schmime. But none of our statements about time connect up with that other block. Only statements about hyper-schmime do. This would raise some questions, including what I mean, and why I brought it up. That is, what does hyper-schmime have to do with anything we were talking about before, which, importantly, did not include hyper-schmime? Similar questions arise here. What does hypertime have to do with the pairs of propositions whose compatibility was at issue? One salient such pair, as we've seen, is

(7) The universe is only a few thousand years old.

(8) The universe is older than a few thousand years.

On HYP, the objector says, (7) is not true, but (8) is. And it's also true that

(9) The universe hyper-was a few thousand years old.

This raises the question of how (9) is relevant to the compatibility of (7) and (8). What does hypertime have to do with time?

The burden is on the defender of HYP and PT to show that hypertime is relevant in a peculiar way: Even though talk of the past doesn't connect up with the hyper-past, it nonetheless has to admit of a reading that makes it about that hyperpast. It's important that (7) is still false on HYP. Yet for HYP to be relevant, it has to provide one way of reading (7). As Hudson notes, theological tradition says that certain events happened. For HYP to be relevant to the threat of incompatibility with a scientific consensus to the effect that they did not, that claim has to prove to be 'neutral between history and hyperhistory' (193). 
The worry is that instead of (epistemically possible) metaphysical compatibility, at best we have ambiguity between obscurity/irrelevance on the one hand, and straightforward incompatibility on the other.

In short, the comparison with familiar sceptical scenarios isn't favourable to HYP: HYP is just as susceptible to Moorean shifts, and rather more susceptible to intelligibility worries.

\section{Another interpretation of the case for PT}

Consider now the second interpretation of the case for HYP: HYP is no less plausible than some other metaphysical views that are live contenders, and in addition, HYP can do useful work in the philosophy of religion (chapters $7 \& 8$ ). On this interpretation, the implication is that those other views are correctly treated as live contenders, and that since HYP is no less plausible, it's at least somewhat plausible. Relevant other such views are dynamic (tensed, A-theoretic) ones, especially the growing block view (on which spacetime grows) and the shrinking block view (on which spacetime shrinks).

Thus understood, the case for PT is largely presupposed by the book, so I will be brief. No doubt 'not everything can be taken up in one book' (96), but it's not entirely clear why the book 'is no place to adjudicate such attempts at wedge-driving' (85), namely attempts to show that HYP is a less plausible metaphysical view than these A-theories. ${ }^{3}$ Suppose the base level of plausibility of dynamic views in general is not that relevant. And suppose we also ignore the B-theoretic alternative, which for all that's been said includes no 'seemingly bizarre commitments' whatsoever (15). ${ }^{4}$ Still, isn't it relevant how HYP compares to these A-theories when it comes to saying e.g., 'what it takes for a world to be temporal, what it takes for a time to be the present, what it takes for time to pass, what the direction of time turns out to be, which propositions express the laws of nature', and 'whether causation is supposed to track history or hyperhistory' (83/192)? Hudson does devote 19 pages to trans-hypertime identity, which is an issue of this kind. But he discusses none of the others, while dedicating two whole chapters to potential pay-offs of HYP within the philosophy of religion. It's hard not to worry that this reflects a methodological stance on which views are espoused more on the basis of what they can do for us than how compelling they are.

But isn't the book merely intended to show that it's worth investigating HYP further, and that the investigation will take place in metaphysics? And doesn't what I'm saying demonstrate precisely this?

\footnotetext{
${ }^{3}$ Perhaps the omission should count against this interpretation, and against separating this interpretation from the first. However, as noted above, the resulting stance would be somewhat selfundermining: if all that matters is whether someone has in fact taken a view seriously, why even try to make views plausible? Why doesn't anything go?

${ }^{4}$ Note that this means the case in support of the more general aim, even as applied to the metaphysics of time, is also largely implicit.
} 
We are certainly in agreement that metaphysics, and philosophy more generally, is highly relevant to many potential and actual conflicts between science and religion. But to block conclusive dismissal of literalism about Genesis by appeal to science, one does have to show that HYP is a live contender (in a way that familiar sceptical hypotheses aren't). And to the extent that the investigation that would show this has yet to take place, one hasn't blocked the dismissal.

of course, the same holds insofar as the investigation is turning out unfavourably. I expressed my main worry about HYP in section 3. Here are a few more reasons not to hold one's breath. I emphasized that HYP includes specific claims about the contents of space-time blocks, which, if the rest of HYP were successful, would shed doubt on claims like (8) (at least on one reading). This in itself lowers plausibility. A related problem is that principles like 'once past, forever past' are jettisoned. That's a cost of a view, something that one strives to avoid (and saying this does not, contra page 84, require a claim to a priori knowledge that the principle holds necessarily). Moreover, while the aim may just be to establish equal plausibility with say, the growing block view, it's worth noting that that view also faces severe difficulties. For one thing, many of its versions do require hypertime (pp. 82-85 are said to argue against this but I'm not sure how), which is not much less problematic there. For another, insofar as the leading edge of the block is a spatially extended global instant, there is a conflict with relativity theory. Finally, even if intelligible and genuinely distinct from the B-theory, A-theories like the growing block view may well be as explanatorily inert and implausible as vitalism in biology (see Callender (forthcoming)).

\section{Conclusion}

I've described why I remain unpersuaded by the case for PT. As mentioned, the book is full of interesting material, a lot of which I haven't discussed. In spite of - or perhaps because of - points of substantial disagreement, I've benefitted greatly from engaging with it. Relatedly, we are united in thinking of analytic philosophy as a valuable and often under-appreciated conversation partner in the science-religion dialogue.

\section{Acknowledgements}

This response was written while I was a member of the Templeton World Charity Foundation project 'Theology, Philosophy of Religion, and the Sciences' (TWCF0062/AB36). Thanks to Ting Cho Lau, copy editor for JAT, for helpful comments on an earlier draft. 


\section{Bibliography}

Callender, C. (forthcoming) What makes time special? Oxford University Press.

Hudson, Hud. 2014. The Fall and Hypertime. Oxford. 